attended a screening visit; 58/129 screen failed (eg, due to deterioration in peak flow, unable to wean off regular asthma medications) leaving 71 randomised $(2.5 \%)$ of total patients invited. Trial 2: similar picture, completed July 2011, extended by 6 months due to slow recruitment. Target to randomise $=80$, target to complete $=68$. Actual completed: $71 / 8398(<1 \%)$ of those invited.

Conclusion Achieving the completion target in randomised controlled trials requires significant administrative support, and the capacity to increase support should difficulties in recruitment be encountered. Closer partnership with primary care practitioners, better access to primary care patient databases and direct contact with potential recruits can overcome this. Loss of potential recruits during the run-in phase needs exploration, and is of significant importance to improve the efficiency of screening to randomisation. Addressing these issues will mean fewer trials are underpowered and hence provide better return for grant awarding bodies.

\section{P206 PSYCHOLOGICAL COMORBIDITY IN VOCAL CORD DYSFUNCTION}

doi:10.1136/thoraxjnl-2011-201054c.206

E Gregson, S Campbell, S Lillie, R Stacey, J Haines, S Fowler, A Vyas. Royal Preston Hospital, Preston, UK

Background Vocal Cord Dysfunction (VCD) is typically reported to affect young females and has been associated with a psychiatric history. We run a multidisciplinary-based service for VCD patients with input from specialist speech and language therapy, physiotherapy and psychology. We investigated the demographics of our patient cohort with VCD, its association with anxiety and depression and whether this affected treatment response.

Methods All patients referred for specialist speech and language therapy at the Royal Preston Hospital Airways Clinic between June 2006 and May 2011 with VCD confirmed by endoscopy were included. During routine clinical care data were collected including demographic details and comorbidities. Patients were also asked to complete the Hospital Anxiety and Depression (HAD) questionnaire. Subjective symptomatic improvement was recorded at patient follow-up visits.

Results A total of 95 patients were eligible for study inclusion: $73.7 \%$ were female with a median age of 53 (17-83) years while men were older at $59(37-80)$ years. Medical comorbidities included asthma (56.8\%), reflux (47.4\%), chronic cough (17.9\%), nasal disease $(16.8 \%)$ and neurological disease $(12.6 \%)$. A history of confirmed psychiatric disease was noted in $38.9 \%$. In 43 patients who completed HAD scores, moderate or severe anxiety was found in $41.8 \%$ and depression in $23.3 \%$. Response to treatment was excellent (67\% showing clinical improvement) and this was independent of medical or psychological comorbidity.

Conclusions Our data suggest that VCD affects a wide range of patients, in terms of age, gender, comorbidities and HAD scores which do not impact on treatment response, as a result of the multidisciplinary approach and this success is comparable to most asthma therapies when patient compliance and education are accounted for. It challenges many previously held concepts and supports a multidisciplinary approach to treating VCD.

\section{P207 DOES THE NIJMEGEN CORRELATE TO THE D12 WHEN USED AS AN OUTCOME MEASURE IN PATIENTS WITH BREATHING PATTERN DYSFUNCTION}

doi:10.1136/thoraxjnl-2011-201054c.207

R Johnston, F Shaw, A Menzies Gow, L Flude. Royal Brompton and Harefield NHS Foundation Trust, London, UK

Introduction Breathing pattern dysfunction (BPD) in patients with and without respiratory disease is linked to disproportionate dysp- noea. Asthma patients in particular have a high prevalence of BPD. The Nijmegen questionnaire $(\mathrm{NQ})$ is a validated outcome measure in patients with no underlying respiratory pathophysiology, not in patients with organic disease. There is no validated tool that can be used to assess the effectiveness of breathing retraining in patients with BPD. The Dyspnoea-12 (D12) questionnaire provides a global score of breathlessness severity and is validated in respiratory disease, but not in patients with BPD. The aim was to correlate the D12 to the NQ and assess its use as an outcome measure for patients with BPD. Pre and post scores were compared to assess the effectiveness of Physiotherapy breathing retraining on patients with BPD referred to our tertiary centre.

Method We included all Physiotherapy referrals to the outpatient department of the Royal Brompton Hospital for BPD between January and July 2011. The D12 and NQ were completed before and after 3 Physiotherapy interventions and respiratory rate was also measured.

Results Initial data were available for 26 patients: mean (SD) age 47.6 yrs (13), 16 (62\%) female, $13(50 \%)$ had a diagnosis of asthma Post intervention data were available for 17 (65\%) patients. Respiratory rate did not correlate with the $\mathrm{NO}$ or D12 in any of the groups. There was a statistically significant correlation between the $\mathrm{NQ}$ and $\mathrm{D} 12$ both pre $(\mathrm{p}=0.0017, \mathrm{R}=0.59)$ and post $(\mathrm{p}=0.0156$, $\mathrm{R}=0.58$ ) intervention. A statistically significant difference pre and post intervention was shown in the $\mathrm{NQ}(\mathrm{p}=0.0242$ mean $(\mathrm{SD})=7.35$ (12.1)) and the D12 ( $\mathrm{p}=0.0127)$.

Conclusion The data suggests that breathing retraining provides both a clinically effective and statistically significant improvement in symptoms of patients with BPD. There was a significant correlation between the D12 and NQ scores in patients referred with $\mathrm{BPD}$. This highlights that the D12 may be an appropriate outcome measure in this patient population.

\section{P208 OBESITY AUGMENTS CIRCULATING NEUTROPHIL LEVELS IN ASTHMA}

doi:10.1136/thoraxjnl-2011-201054c.208

M C Pynn, G Davies, C A Thornton, A Bryant, R Jones. Swansea University, Swansea, Wales

Introduction Mechanisms underlying the association between obesity and asthma are not well understood. Obesity is characterised by chronic inflammation and adipose tissue, comprising up to $50 \%$ pro-inflammatory cells, produces many pro-inflammatory cytokines and hormones (adipokines). Enhanced systemic inflammation might provide the causal link between obesity and asthma. Although there are good mechanistic data that obesity can augment innate immune function and promote immune dysregulation by reducing regulatory $\mathrm{T}$ cell (Treg) numbers there is little work in this area in relation to asthma.

Methods A case-control study is being conducted examining six groups of pre-menopausal women $(n=120)$ : normal weight (BMI $20-25 \mathrm{~kg} / \mathrm{m}^{2}$ ), overweight (BMI $25-30 \mathrm{~kg} / \mathrm{m}^{2}$ ) and obese individuals $\left(B M I>30 \mathrm{~kg} / \mathrm{m}^{2}\right)$ with and without asthma. Asthma diagnosis was physician confirmed, and severity graded. Measures of adiposity, lung function and blood were collected during menstruation. Automated haematology analysis was used to quantify major cell types and chemiluminesence to measure whole blood reactive oxygen species generation following stimulation. Flow cytometry was used to examine major lymphocyte subtypes including Treg cells. A number of circulating cytokines and adipokines will be measured on sample collection completion.

Results Interim analysis of 36 individuals revealed a significant increase in circulating total leucocytes with increasing BMI which is more pronounced in asthmatics compared with controls $(p=0.022)$ This appears to be due to a significant increase in neutrophils 
(Abstract P208 figure 1, $(\mathrm{p}=0.018)$ ). In parallel, reactive oxygen species generation increased with each BMI category $(p=0.026)$. Furthermore, the eosinophil count dropped significantly with increasing BMI in the asthma patients ( $\mathrm{p}=0.045$ ).

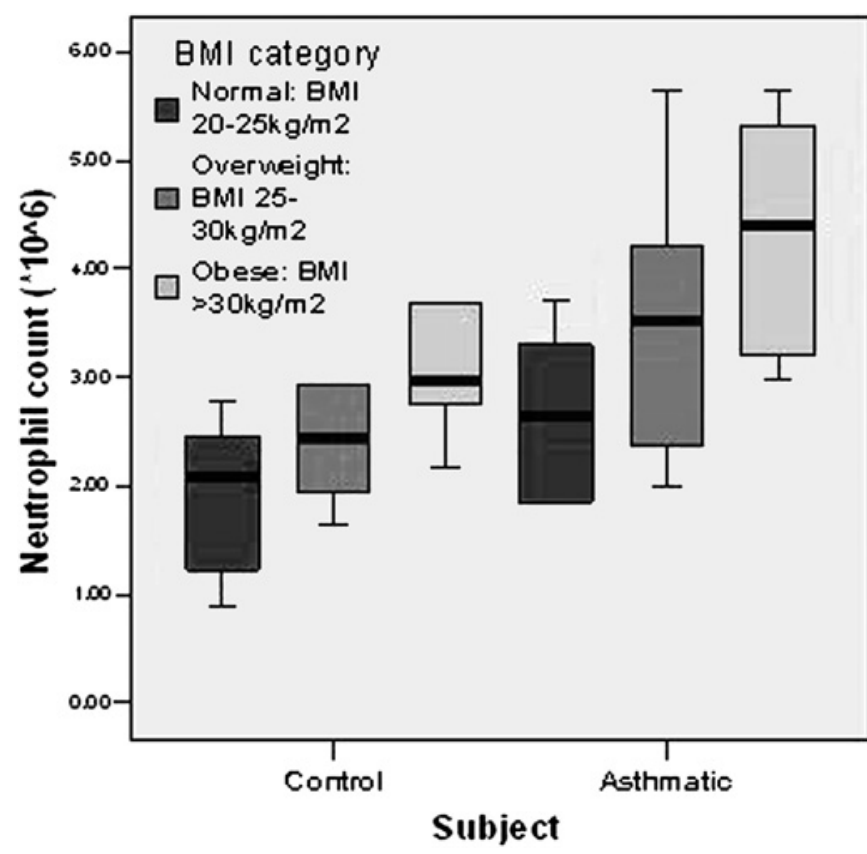

Abstract P208 Figure 1 Whole blood neutrophil count in controls vs asthmatic individuals according to BMl category $(p=0.022)$.

Conclusion Preliminary data from this study suggests that obesity is associated with systemic inflammation resulting in increasing levels of circulating neutrophils and that this is more marked in asthmatics than controls. This is in keeping with recent work showing increased neutrophils locally in the sputum of obese asthmatics. Our findings could explain the reduced inhaled corticosteroid efficacy observed in this asthma phenotype.

\section{COPD: exacerbations, survival and end of life care \\ P209 RISK FACTORS FOR RESPIRATORY EXACERBATIONS IN THE BOLD STUDY}

doi:10.1136/thoraxjnl-2011-201054c.209

${ }^{1} \mathrm{~L}$ Gnatiuc, ${ }^{1} \mathrm{~S}$ Coton, ${ }^{2} \mathrm{~S}$ Buist, ${ }^{1} \mathrm{~B}$ Kato, ${ }^{3} \mathrm{P}$ Mejza, ${ }^{1} \mathrm{P}$ Burney. ${ }^{1} / \mathrm{mperial}$ College London, London, UK; ${ }^{2}$ Oregon Health \& Sciences University, Portland, USA; ${ }^{3}$ Jagiellonian University School of Medicine, Cracow, Poland

Background Exacerbations are important clinical events related to long-term outcomes in asthma and Chronic Obstructive Pulmonary Disease (COPD). However their determinants are not fully understood (Hurst et al, 2010). We used the BOLD data to assess the prevalence and risk factors for respiratory exacerbations among population-based samples of adults aged $40+$ across 18 BOLD sites. Methods Exacerbations were defined as at least one reported episode of breathing problems that interfered with usual daily activities or led to missing work in the previous 12 months. Multiple logistic regressions were used to estimate the effects of potential risk factors for reported exacerbations: medical history, FVC, occupational, biomass and smoking exposures, education, age and sex. All effects were mutually adjusted and estimated using probability weights to allow for the sampling design. Regression models were fitted separately for each centre before pooling results across centres using random effects meta-analysis. Heterogeneity was summarised using the $\mathrm{I}^{2}$ statistic.

Results The 12-month prevalence of reported exacerbations ranged from $1.9 \%$ in Guangzhou, China to $14.2 \%$ in Lexington, USA, it was higher in subjects with spirometrically defined COPD as compared to subjects without spirometric COPD $(14.4 \%$ vs $4.2 \%, \mathrm{p}<0.0001)$ and in low and middle income countries as compared to high income countries $(7.9 \%$ vs $4.9 \%, p<0.0001)$. Exacerbations were associated with doctor diagnosed asthma, COPD stage $1+$, chronic bronchitis, increase in MMRC dyspnoea score, current exposures to biomass and dusty jobs and history of TB (see Abstract P209 table 1). Similar trends for overall effect estimates were obtained for low and middle income countries and high income countries with and without biomass exposure information. The variation in reported exacerbations across sites is unlikely to be due to variation in the influence of different risk factors between sites except for COPD stages 1 and $2\left(I^{2}=44 \%, p=0.03\right)$, biomass exposure $\left(I^{2}=57 \%\right.$, $\mathrm{p}=0.01)$ and reported history of $\mathrm{TB}\left(\mathrm{I}^{2}=65.2 \%, \mathrm{p}=0.001\right)$.

Abstract P209 Table 1 Risk factors for exacerbations (meta-analysis)

\begin{tabular}{|c|c|c|c|}
\hline & $\begin{array}{l}\text { Overall Adjusted OR* } \\
(95 \% \mathrm{CI})\end{array}$ & $\begin{array}{l}\text { Overall } I^{2} \\
(\%)\end{array}$ & $\begin{array}{l}\text { p Value for } \\
\text { overall } I^{2}\end{array}$ \\
\hline Female & 1.45 (1.00 to 2.09$)$ & 0.0 & 0.8 \\
\hline Age & $0.97(0.95$ to 0.99$)$ & 42.1 & 0.03 \\
\hline Education level & $0.95(0.78$ to 1.15$)$ & 18.6 & 0.2 \\
\hline Pack years smoked-per 10 increase & $0.90(0.73$ to 1.12$)$ & 24.7 & 0.2 \\
\hline Ex-smoker & $1.19(0.81$ to 1.74$)$ & 0.0 & 0.8 \\
\hline Current-smoker & $1.37(0.85$ to 2.21$)$ & 0.0 & 0.5 \\
\hline COPD—mild and moderate & 2.55 (1.70 to 3.82$)$ & 44.0 & 0.03 \\
\hline COPD—-severe and very severe & $3.62(2.11$ to 6.18$)$ & 0.0 & 0.6 \\
\hline Doctor Diagnosed Asthma & 4.92 (3.77 to 6.42$)$ & 0.0 & 0.9 \\
\hline MMRC Dyspnoea-increased severity & 2.14 (1.86 to 2.46$)$ & 31.7 & 0.1 \\
\hline Chronic bronchitis & 3.03 (1.97 to 4.66$)$ & 14.4 & 0.3 \\
\hline Post BD FVC & $0.90(0.68$ to 1.19$)$ & 40.4 & 0.04 \\
\hline Current biomass exposure & 2.67 (1.25 to 5.68$)$ & 57.0 & 0.01 \\
\hline Current dusty job & 1.60 (1.16 to 2.22$)$ & 15.5 & 0.3 \\
\hline CVD & 1.28 (0.98 to 1.68$)$ & 0.0 & 0.9 \\
\hline Diabetes & $1.52(0.94$ to 2.48$)$ & 35.3 & 0.08 \\
\hline Tuberculosis & $2.76(1.10$ to 6.91$)$ & 65.2 & 0.001 \\
\hline Childhood respiratory disease & $1.52(0.92$ to 2.51$)$ & 0.0 & 0.6 \\
\hline Family history of COPD or asthma & $1.35(1.00$ to 1.83$)$ & 11.4 & 0.3 \\
\hline
\end{tabular}

${ }^{*}$ Mutual adjustment for all the risk factors in the table and height and weight, weighted for survey design.

Conclusion Respiratory exacerbations commonly occur in those with normal ventilatory function. Exacerbations are associated with severity of COPD, doctor diagnosed asthma, and environmental exposure to biomass and workplace dust. Chronic cough and phlegm and a diagnosis of emphysema are also associated with an increased risk for exacerbation in people with and without irreversible airway obstruction.

\section{P210 WHICH SYMPTOMS PROMPT PATIENTS WITH AN EXACERBATION OF COPD TO PERSEVERE, SELF-TREAT OR SEEK CARE? A COMPARISON WITH HEALTH PROFESSIONALS}

doi:10.1136/thoraxjnl-2011-201054c.210

1J S Wilson, ${ }^{2} \mathrm{~J} J$ Sittlington, ${ }^{2} \mathrm{E}$ McCrum-Gardner, ${ }^{3} \mathrm{~F}$ Kee, ${ }^{1} \mathrm{~J} S \mathrm{~S}$ Elborn. ${ }^{1}$ Belfast Heath \& Social Care Trust, Belfast, Northern Ireland; ${ }^{2}$ University of Ulster, Jordanstown, Northern Ireland; ${ }^{3}$ Queen's University, Belfast, Northern Ireland

Introduction and Objectives Exacerbations of Chronic Obstructive Pulmonary Disease are a major cause of morbidity and mortality. Despite the increased healthcare utilisation involved in treatment, 